\title{
La enseñanza de la comunicación: una mirada a los enfoques teóricos y metodológicos de profesores y programas de teorías de comunicación en Bogotá*
}

The Teaching of Communication: A Look to the Theoretical and Methodological Approaches of Teachers and Programs on the Communication Theory in Bogotá

O ensino da comunicação: um olhar aos enfoques teóricos e metodológicos de professores e programas de teorias de comunicação em Bogotá

Claudia Pilar García Corredor ${ }^{\text {a }}$

Pontificia Universidad Javeriana, Colombia

pigarcia@javeriana.edu.co

ORCID: http://orcid.org/0000-0001-8873-5762

Ángela Marcela Castellanos Barbosa

Pontificia Universidad Javeriana, Colombia

José Miguel Pereira González

Pontificia Universidad Javeriana, Colombia

ORCID: http://orcid.org/0000-0003-4970-1839
DOI: https://doi.org/10.11144/Javeriana.syp38-75.ecme

Fecha de recepción: 20 Junio 2017

Fecha de aprobación: 18 Enero 2019

Fecha de publicación: 15 Noviembre 2019

\section{Resumen:}

A la luz de las teorías que iluminan el campo y los enfoques pedagógicos, el presente texto se ocupa de hacer una mirada a la enseñanza de las teorías de comunicación en diferentes facultades de comunicación de Bogotá. Es claro que en todo proceso de enseñanza-aprendizaje, un actor fundamental es el estudiante; sin embargo, la investigación aborda la tensión entre dos sujetos del proceso: los maestros y los programas de teorías de comunicación.

Palabras clave: teorías y metodologías de comunicación, pedagogías de comunicación, enfoques pedagógicos de comunicación.

\section{Abstract:}

The light shed by theories dealing with this issue and the related pedagogical approaches inform this paper, which is intended as a look to the teaching of the communication theories in different communication colleges in Bogotá. It is clear that in any teachinglearning process the student is a fundamental actor; however, the research deals with the tension between two subjects in the process: teachers and programs of communication theories.

Keywords: communication theory and methodologies, communication pedagogies, pedagogical approaches of communication.

\section{Resumo:}

À luz de teorias que iluminam o campo e os enfoques pedagógicos, o presente texto faz um olhar ao ensino das teorias de comunicação em diferentes faculdades de comunicação de Bogotá. É claro que em todo processo de ensino-aprendizagem, um ator fundamental é o aluno. No entanto, a pesquisa aborda a tensão entre dois sujeitos do processo: os docentes e os programas de teorias de comunicação.

Palavras-chave: teorias e metodologias de comunicação, pedagogias de comunicação, enfoques pedagógicos de comunicação.

La educación es un arte, cuya práctica

ha de ser perfeccionada por muchas generaciones.

Fuente: Kant

Notas de autor:

a Autora de correspondencia. Correo electrónico: pigarcia@javeriana.edu.co 


\section{Introducción}

La noción de teorías de comunicación, así como los distintos debates alrededor de esta, motivan una reflexión en torno a las categorías cómplices en los procesos didácticos, pedagógicos y metodológicos de la enseñanza. El tema está abierto y es propicio para la investigación y el debate académico. Cabe recordar que hacia finales de los años cincuenta, Berelson (2004), al referirse a la inminente "muerte" del campo, sostenía la necesidad de explorar líneas de investigación en comunicación, con una preocupación epistemológica, para evitar las repeticiones de las aproximaciones realizadas en la primera parte del siglo XX. Es preciso afirmar desde Fuentes Navarro que las "teorías de la comunicación" deben ser comunicadas para ser tales, y el proceso de comunicarlas entre sujetos sociales concretos en entornos concretos, afecta de alguna manera su naturaleza meta- comunicativa. También viceversa: "Los modelos teóricos de la comunicación afectan de alguna manera la práctica comunicativa” (Vidales, 2010, pp.7-8). Por ello, resulta pertinente la relación planteada, en tanto la práctica de la enseñanza-aprendizaje de las teorías es un acto comunicativo de doble vía.

En el texto ¿Para qué sirve estudiar Teorías de la Comunicación?, Manuel Martín Serrano plantea una abierta provocación a expandir la mirada sobre la comunicación desde la pregunta que funda el estudio científico de la misma: “Cómo es (a veces) posible que la comunicación sea posible? o alternativamente: ¿cómo es posible (a veces) que la comunicación no sea posible?” (Martín Serrano, 2006, p. 45) Desde la perspectiva de este autor, la teoría de la comuniación se encarga de indagar por las formas de producción, reproducción y circulación de la información para la transformación de las poblaciones, de manera que la enseñanza misma de la comunicación es un acto comunicativo objeto de estudio de las teorías que se enseñan. En este sentiodo, vale la pena recordar que para Martín Serrano, la "teoría no se contrapone a práctica. Toda actividad socialmente organizada, incluida la práctica profesional de la comunicación, aplica alguna teoría, aunque no esté explícita o sea falsa" (Martín Serrano, 2006, p. 45). Estas afirmaciones motivan, entonces, la reflexión acerca de las complicidades de las que se vale el profesor para los procesos de enseñanza de las teorías sobre la comunicación.

Es preciso identificar que los debates sobre el estudio de la comunicación han pasado por quienes, como Bernard Berelson, en su momento consideraban que este campo moría, o por aquellos -como Wilbur, Schramm, Riesman y Baver- que señalaban que este campo apenas estaba naciendo (Vidales Gonzáles, 2011). La propuesta es identificar qué viene sucediendo con el estudio de la comunicación desde la enseñanza de las teorías que la abordan como campo de saber.

Esta exploración se pretende como un desafío para quienes asumen la tarea académica de comprender y enseñar la comunicación, fundamentalmente porque hablar de este campo implica un acto comunicativo; de manera que dichas teorías, para ser enseñadas, deben ser comunicadas desde modelos teóricos que determinen la práctica comunicativa y educativa.

En relación con la enseñanza de las teorías de comunicación, Marta Rizo (2012) plantea la pregunta “¿cómo generar una formación profesional clara y coherente, acorde con las particularidades del campo, que articule las diferentes vertientes de la comunicación, y que, en el mejor de los casos, responda a las demandas formativas del actual escenario comunicativo?” (p. 13). Es desde la Edad Media en Europa que educar significa disciplinar, adquirir conocimientos a partir del aprendizaje de habilidades, con el propósito de generar prácticas desde la obediencia, como una acción sometida para corregir y disciplinar. En la Ilustración se establecen prácticas a partir de la racionalidad y se perfila como objetivo generar procesos de aprendizaje desde un accionar racional con la idea del adiestramiento. Otra intención formativa durante el siglo XX tiene el propósito de educar para pensar y para hacerse preguntas, se prepara para la crítica y se privilegia el planteamiento de las preguntas para conocer, en vez de para obtener respuestas absolutas, postura que se fundamenta en autores de la pedagogía crítica, como Paulo Freire y Mario Kaplún, entre otros pensadores latinoamericanos. 
Este trabajo recoge lo que enuncian los profesores de asignaturas de teorías de comunicación en relación con lo que muestran los syllabus para entender cómo se enseñan dichas teorías a partir de los enfoques educativos, entendidos estos como modos del acto educativo y de su configuración en prácticas cotidianas de enseñanzaaprendizaje.

Para el análisis se tuvieron en cuenta los syllabus de las asignaturas de teorías de la comunicación. El syllabus es un documento considerado como un micro currículo en el que se solicita la descripción, el propósito, los objetivos específicos, los materiales y recursos de todo tipo, el cronograma por semana, las formas de evaluación y las normas del curso. Esta es una descripción general que varía de una institución a otra; sin embargo, siempre el syllabus, por definición, trata de especificar los objetivos, la tabla de contenidos, los métodos, los medios, los recursos que se emplean y las evidencias de aprendizajes al finalizar el curso. Se presenta como una herramienta que permite que el estudiante identifique información general del curso, y pueda prever lo que el profesor espera de él. Se concibe como una evidencia de la calidad de la enseñanza y el aprendizaje que se va a dar a lo largo de un curso, mas no en todos los casos se articula con los objetivos de la carrera y de la universidad; por lo tanto, se ha utilizado como una matriz que entregan las decanaturas o direcciones de programas académicos para que el profesor lo tome de base para realizar su curso. Así, el syllabus cumple un papel de gran importancia y se concibe como el documento que establece el primer contacto entre el estudiante y el docente, el cual cumple con las funciones de establecer el tono del curso y la docencia, definir las responsabilidades del profesor y de los estudiantes en su aprendizaje activo, y orientar a los estudiantes en su nivel de preparación para tomar el curso. En términos generales, describe los recursos de aprendizajeenseñanza.

\section{De los enfoques teóricos de la comunicación, enfoques pedagógicos y rol del profesor: un horizonte conceptual}

Abordar el campo de estudios de la comunicación no es una tarea reciente. Desde comienzos del siglo $\mathrm{XX}$, se materializa este análisis en la configuración naciente de un campo de estudios, que, como menciona Mattelart en el libro "La Historia de las Teorías de la Comunicación", es una polifonía, y en el contexto de "la proliferación de las tecnologías y la profesionalización de las prácticas no han hecho sino sumar nuevas voces a esta polifonía en un final de siglo que hace de la comunicación la figura emblemática de las sociedades del tercer milenio" (Mattelart, 1997, p. 9).

Se identifica que las variaciones que va sufriendo el concepto de comunicación corresponden con una visión de mundo que varía de acuerdo a los intereses de la época. En palabras de Serrano (como se citó en Mattelart, 1997):

La clase dominante en cada sociedad procura imponer al conjunto de la comunidad una explicación de la naturaleza de la sociedad, de la cultura, que sirva a sus intereses y contribuya a la perpetuación del orden establecido... En la ciencia, el arte, la religión, la educación, la información, la comunicación, se encuentran los modelos ideológicos que legitiman el dominio. Por ejemplo, la dependencia social de los grupos marginados (negros, chicanos, gitanos) se «explica» ideológicamente por aquella ciencia psicológica que les suponga menos inteligentes, por aquella ética que les considere menos «morales», por aquel cine que les presente en papeles de delincuentes, ladrones, drogadictos, etc. (p. 196)

La comunicación como campo de conocimiento se nutre, desde el surgimiento de su conceptualización a comienzos del siglo XX, de diferentes otros saberes, como la psicología, la filosofía, la lingüística y la antropología, situación que explica los componentes de interés multidisciplinar.

Los trabajos teóricos de Alsina (2001), de Moragas (2011), Fuentes Navarro y Vasallo de Lopes (2001), Galindo (2008), Martino (2007), Mattelart (1997), Rizo (2012), Torrico (2004, 2010) y Saintout (2003), coinciden en los principales abordajes y los enfoques que han constituido las discusiones en el proceso de construcción del campo de estudios de la comunicación. El planteamiento de Eric Torrico (2010) sostiene 
que las matrices teórico-sociales, estructural-funcionalista, la dialéctica crítica, el estructuralismo, la teoría de sistemas, el posestructuralismo, entre otras, permiten identificar, al menos, cuatro maneras de estudiar la comunicación: pragmático, socio-técnico, crítico y político-cultural. A partir de estos abordajes, y para efectos del análisis de los syllabus de los programas y cursos de teorías seleccionados para este estudio, se decidió desagregar la propuesta del autor y trabajar con los siguientes enfoques teóricos de comunicación.

Por un lado, el enfoque funcionalista comprende las perspectivas que centran el interés en la transmisión eficaz de mensajes, generación de efectos, cumplimiento de funciones de los medios, influencia personal, presión grupal, difusión de innovaciones, los usos y gratificaciones, establecimiento de agenda, la espiral del silencio, la cultura de masas, el determinismo tecnológico, entre otros. El enfoque estructuralista incluye la perspectiva que hace énfasis e indaga sobre el mensaje y sus significados, por sus estructuras significantes, y se alimenta de los estudios de la lingüística, la semiología y la semiótica. Así mismo, se ocupa del estudio de los textos, los lenguajes, las narrativas y los discursos comunicativos. El enfoque crítico comprende la visión que centra su atención en las preguntas por la ideología dominante, la construcción de hegemonía, las estructuras significantes, los aparatos ideológicos, la industria cultural, la dependencia cultural, el imperialismo cultural, las micro-resistencias, la economía política de la información y la comunicación, el nuevo orden de la información, el alternativismo y la comunicación popular. El enfoque culturalista incluye los programas que hacen énfasis en los estudios culturales, las mediaciones, las prácticas de comunicación, la recepción crítica, el consumo, usos y apropiación de los medios, el consumo cultural, los frentes culturales, la mediología, estudios decoloniales y los estudios posmodernos. El enfoque pragmático comprende la perspectiva que hace énfasis en la comunicación humana, la comunicación interpersonal, la interacción simbólica, la vida cotidiana y la sistémica mediática; y, por último, el enfoque sociotécnico incluye a aquellos programas que centran su atención en los estudios sobre la ciencia, la tecnología y la sociedad, la ecología de medios, la mediología, las ciberculturas, el determinismo tecnológico, la ecología de los medios y los ecosistemas comunicativos.

Por otro lado, se contemplan cuatro enfoques pedagógicos, en relación con las pedagogías, las metodologías y las didácticas que dieron foco a la observación en cuanto a objetivos, estrategias pedagógicas, criterios y/o aspectos que se evalúan en los syllabus; y se retoman algunos autores que aportan a esta caracterización: de Zubiría (2008) y Flórez (2008).

El enfoque pedagógico tradicional concibe la relación maestro-alumno como una jerárquica, con un marcado acento vertical, en la cual el maestro sabe y el alumno es una tabula rasa. El texto escolar es el centro del aprendizaje y se constituye en un texto dogmático escrito por una autoridad. Bajo esta perspectiva, los procesos cognitivos del aprendizaje se centran en la memorización, no en el debate ni en la construcción colectiva. El enfoque pedagógico conductista propone que el sujeto logre cambios en la conducta, y funciona a partir de estímulos, como premio o castigo, para generar una respuesta prevista por el profesor. En este enfoque hay una respuesta pasiva y automática a estímulos externos, es un aprendizaje pasivo y no negociado basado en métodos objetivantes. El Enfoque sociocultural tiene en cuenta el contexto y prioriza el resultado de la interacción del individuo con su entorno. En el enfoque pedagógico constructivista, el sujeto cognoscente desempeña un papel activo y colaborativo en el proceso de construcción del conocimiento. El aprendizaje se da mediante la experiencia.

Los enfoques pedagógicos inciden en la formación de los estudiantes y en la relación profesor-alumno, inquietudes que han sido enriquecidas por colegas mexicanos, entre ellos Marta Rizo y, recientemente, Carlos Vidales, sin desconocer el valioso aporte de otros investigadores latinoamericanos desde distintas latitudes. En ese contexto, la reflexión sobre aquello que Rizo (2012) denomina "el arduo trabajo de reflexión sobre la formación en comunicación" (p. 15), alude tanto a las incidencias para el campo mismo de la comunicación, como también al enfoque como práctica educativa.

Para pensar el rol del docente se tendrá en cuenta la perspectiva kantiana, desde la cual se entiende el proceso educativo como propio de los hombres. "Únicamente por la educación el hombre puede llegar a ser hombre. No es, sino lo que la educación le hace ser. Se ha de observar que el hombre no es educado más que por 
hombres, que igualmente están educados" (Kant, s. f., p. 3), lo cual pone de manifiesto que el papel del profesor es relevante en la configuración del ser.

Acerca de rol del profesor, se tiene en cuenta que, como dice Kant (s. f.),

al hombre se le puede adiestrar, amaestrar, instruir mecánicamente o realmente ilustrarle. Se adiestra a los caballos, a los perros, y también se puede adiestrar a los hombres. Sin embargo, no basta con el adiestramiento; lo que, importa, sobre todo, es que el niño aprenda a pensar. (p. 6)

Lo anterior implica un proceso de discernimiento como consecuencia de atreverse a pensar para construir conocimiento propio. En este sentido, la pregunta es ¿en la enseñanza de teorías de la comunicación se enseña a pensar para comprender el fenómeno de la comunicación? ¿se enseña a repetir teorías como fundamento obligado? $\mathrm{O}$, por otro lado, ¿las teorías sirven para pensar y ser sujeto en formación?

En la pertinencia de la investigación, Kant (s. f.) plantea la diferencia entre el profesor-instructor y el profesor-director, "nace la diferencia entre el instructor (Infórmator), que es simplemente un profesor, y el ayo (Hofmeaster), que es un director. Aquél educa sólo para la escuela; este, para la vida” (p. 7). Así, el educador está abocado a formar, tanto en la dimensión mecánico-escolástica a la que se refiere el autor -la habilidad la cual constituye al profesor en un instructor-, como en la dimensión para la vida a la que él denomina la prudencia, que configura al profesor en el guía que lleva al aprendiz al proceso de constituirse en hombre para lo público, es decir en ciudadano. En este ejercicio de investigación se pretende reconocer la forma en que aparecen los profesores en la enseñanza de las teorías de comunicación.

\section{Proceso metodológico}

La investigación aquí referida comprende el entrecruce entre dos criterios de análisis: enfoques educativos y tipos de cursos. El estudio se centró en el análisis de los syllabus de Comunicación Social y/o Periodismo de 16 universidades ${ }^{i}$ de Bogotá, con 21 programas, de los cuales se revisaron 114 asignaturas del componente de teorías de la comunicación, de los cuales 108 son de formación presencial. De igual manera, se caracterizó el perfil de los profesores mediante la aplicación de una encuesta en las universidades seleccionadas, se realizaron seminarios de profesores con expertos académicos en el tema, y se desarrolló un taller de devolución creativa en la perspectiva de la metodología de la Investigación Acción Participativa (IAP), propuesta por el sociólogo colombiano Orlando Fals Borda (2008), quien legitima el diálogo como posibilidad para el debate y la reflexión en el escenario de la investigación cualitativa.

La definición de las asignaturas de los diferentes programas se estableció de la siguiente manera: revisión de los planes de estudio de 21 programas, preselección del grupo de asignaturas consideradas teóricas en cada programa ; validación de la preselección con decanos, directores y/o coordinadores de programa en las distintas universidades, a fin de establecer la muestra definitiva de asignaturas con componente teórico ; selección de las asignaturas definitivas para el proceso de análisis, recolección de los respectivos syllabus o contenidos analíticos de las asignaturas seleccionadas; diseño y validación de una matriz para el registro de los datos - a partir de la institución, la facultad, el programa, el nombre, la articulación con el plan de estudios , el semestre en el que se ofrece, el número de créditos, las horas semanales, el pre-requisito, la justificación, el objetivo general , los objetivos específicos, los ejes temáticos o contenidos, el componente-eje-área y la bibliografía - y el diligenciamiento y procesamiento de la matriz para la fase de análisis de los 114 syllabus .

Se siguió un camino inductivo y comprensivo, el cual. según Joseph Maxwell (2004), es uno de los rasgos más destacados de la investigación cualitativa, en conjunto con el interés por el significado y la interpretación, el énfasis en contexto y los procesos. Para ello, se aprovechó, tanto la experiencia y los datos empíricos, como el andamiaje teórico, lo que permitió el siguiente proceso: a) reconstrucción del concepto general de teoría y de las teorías de la comunicación , b) revisión de los datos empíricos (los contenidos de los syllabus y algunos proyectos educativos de los programas académicos) para establecer categorías emergentes; c) realización de 
dos seminarios para profundizar en la construcción conceptual de los debates contemporáneos sobre las teorías y la enseñanza de las teorías de la comunicación; el primero con el profesor brasilero, Luiz Martino, y el segundo con el profesor mexicano, Raúl Fuentes Navarro. Adicionalmente, se establecieron conversaciones con el profesor Erick Torrico . d) Construcción de una matriz centrada en los enfoques pedagógicos que enunciaban los syllabus de las asignaturas elegidas como muestra, la cual se aplica a 108 syllabus que corresponden a los programas presenciales abordados en la investigación, y e) la realización de un taller de devolución creativa con profesores de teorías de comunicación de universidades de Bogotá.

Los criterios del análisis del componente pedagógico son los enfoques educativos y los tipos de cursos, los cuales se sistematizan en una matriz que contiene los cuatro enfoques educativos: tradicional, conductista, sociocultural y constructivista, en relación con los tres tipos de cursos: especificos sobre la comunicación, bistoriográficos y cursos articulados a los oficios, los cuales se observan según los objetivos, las estrategias pedagógicas y los aspectos a evaluar que los syllabus registran. Así, se identifican unas tipologías de asignaturas que responden a la siguiente clasificación (tabla 1 ):

TABLA 1

Tipologías de asignaturas

\begin{tabular}{ll}
\hline Tipologías de asignaturas & Porcentaje \\
\hline Cursos historiográficos & $36 \%$ \\
\hline Cursos sobre conceptos específicos & $30 \%$ \\
\hline Cursos articulados a los oficios y/o profesiones & $34 \%$ \\
\hline
\end{tabular}

Fuente: elaboración propia

Cursos historiográficos: son aquellos cursos que refieren de manera cronológica a autores, paradigmas y enfoques sobre los estudios de la comunicación. Son asignaturas que abordan tanto los paradigmas clásicos, como los debates contemporáneos de la comunicación. En ellos se realizan balances y panoramas en torno al campo de estudios de la comunicación. La figura 1 muestra los cursos historiográficos por institución.

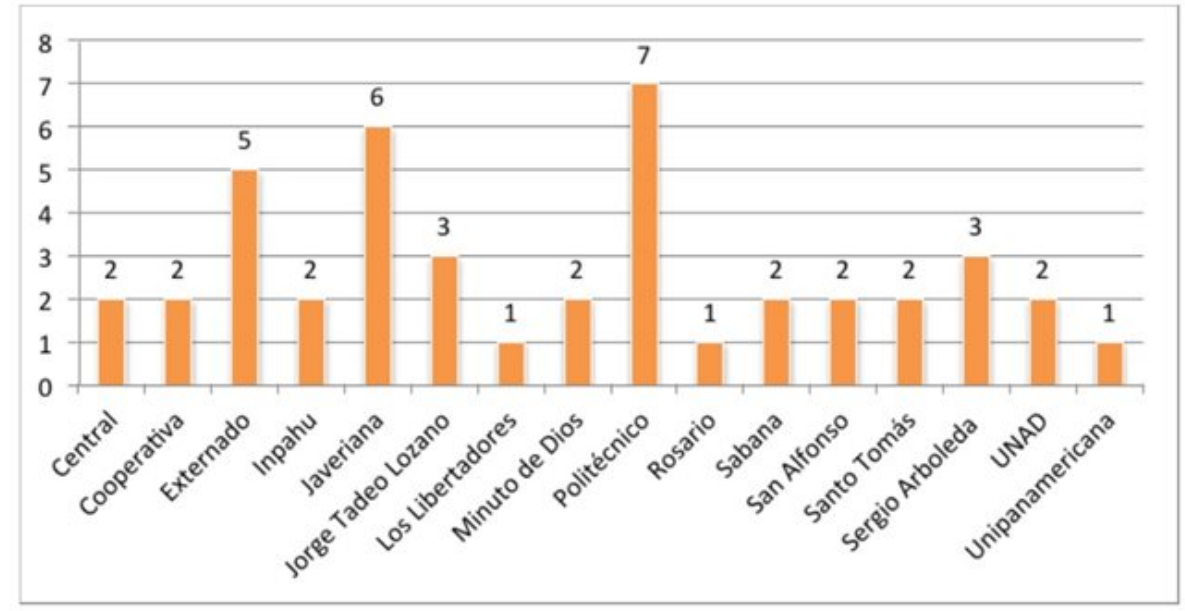

FIGURA 1

Cursos historiográficos por institución

Fuente: elaboración propia

Cursos sobre conceptos especificos: estos se entienden como aquellos que abordan las categorías o los conceptos específicos sobre la comunicación, los cuales buscan desarrollar la perspectiva del plan de estudios o hacer una profundización en un concepto propio de las ciencias sociales o humanas, y la 
comunicación, dentro de una perspectiva interdisciplinar. Algunos ejemplos puntuales son los siguientes: acción colectiva, mediación, opinión pública, cambio social, periodismo, desarrollo, vida cotidiana, interactividad, globalización, cultura, poder. En la figura 2 se exponen los cursos sobre conceptos específicos por institución.

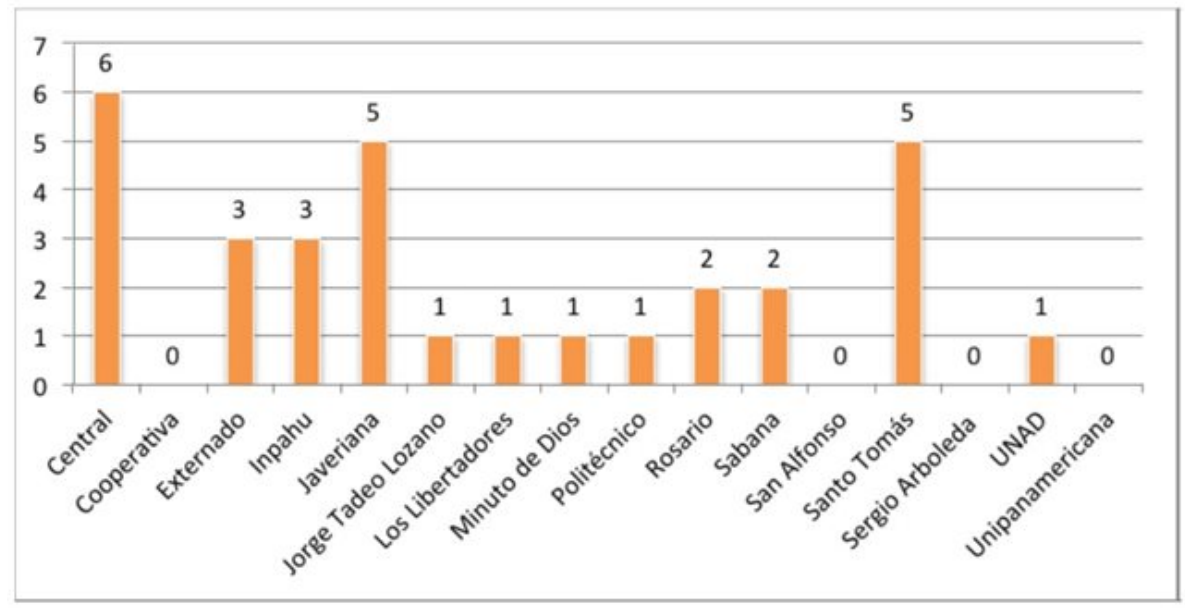

FIGURA 2

Cursos sobre conceptos específicos por institución Fuente: elaboración propia

Cursos articulados a los oficios y/o las profesiones: son aquellos que abordan la especificidad de los énfasis o áreas profesionales, y que se perciben como desarrollos teóricos propios de las profesiones o aplicaciones de la comunicación. Algunos ejemplos son: comunicación organizacional, teorías del periodismo, comunicación publicitaria, comunicación audiovisual, entre otros. A continuación, se muestran los cursos articulados a los oficios y/o las profesiones por institución (figura 3).

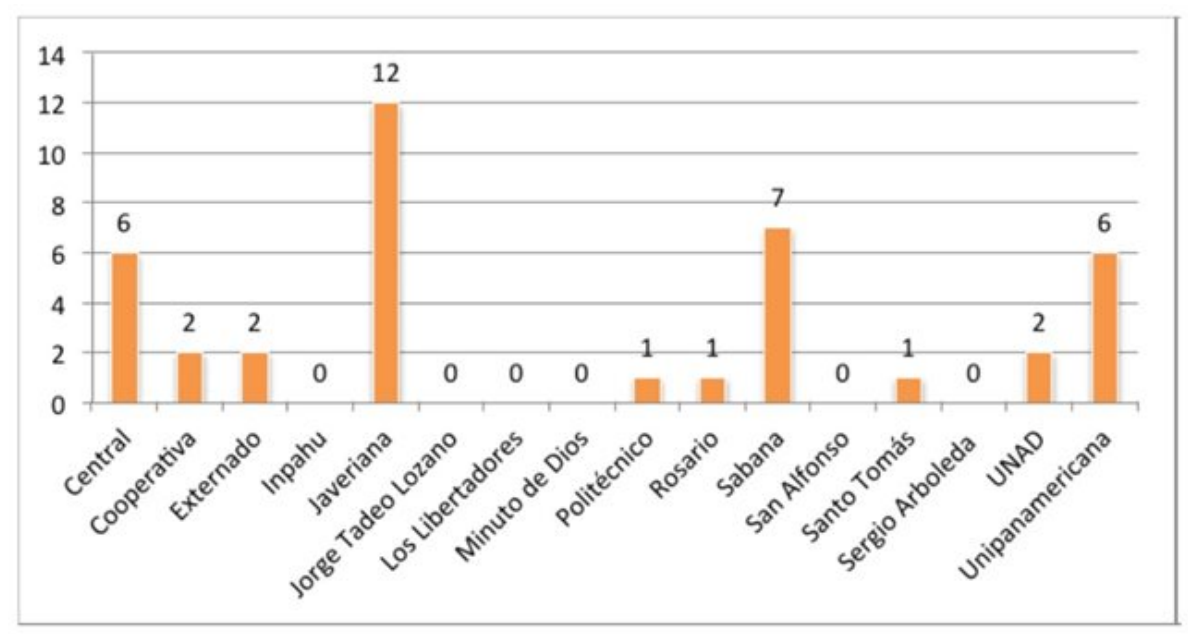

FIGURA 3

Cursos articulados a los oficios y/o las profesiones por institución

Fuente: elaboración propia

En primer lugar, la investigación permite develar, a partir de los syllabus investigados, una serie de hallazgos respecto a la enseñanza sobre teorías de la comunicación, a manera de provocación para futuras investigaciones; y en segundo lugar, sugerir algunas ideas sobre la futura producción teórica en y sobre comunicación, en relación con las tendencias más relevantes sobre este asunto en la segunda década del nuevo milenio. 


\section{Algunos de los hallazgos}

Se presentan los hallazgos del estudio a partir de lo identificado en el taller de devolución creativa, los seminarios con profesores, los syllabus y las encuestas.

El taller de devolución creativa con los profesores permitió identificar tres preocupaciones presentes: cómo enseñar, qué enseñar y para qué enseñar. El cómo está sintonizado con lo que se encontró en los syllabus, es decir, con una gran variedad de metodologías que van desde una preocupación por el proceso hasta los actos más tradicionales en una evaluación que premia y castiga. El qué y el para qué generaron un debate que lleva a los profesores a reflexionar sobre las directrices institucionales que promueven la práctica de los diversos oficios de la comunicación (audiovisual, periodismo, organizacional, editorial, publicidad, radio, entre otros), hasta la comprensión del campo desde la perspectiva epistemológica y como fenómeno social y cultural a la luz de la diversidad de los autores emblemáticos de las teorías de la comunicación.

Lejos de concluir que resulta imprecisa la noción de teorías de la comunicación, la diversa cantidad de enfoques, los conceptos y las categorías abordadas y articuladas a los diseños

curriculares, y los oficios y aplicaciones que se hacen de la comunicación, resultan ser un valor agregado que da cuenta de la vitalidad que tiene el campo, el cual se mueve en una tensión entre la riqueza y la diversidad conceptual -inter o transdisciplinaria-, y la diversidad de estudios específicos hacia los oficios y profesiones de la comunicación.

Se constata que cada programa diseña sus cursos de acuerdo a la filosofía institucional, su misión, visión y apuestas formativas, lo cual determina la orientación central del propio enfoque curricular.

El análisis, particularmente a partir del taller de devolución creativa, permite inferir un desfase entre los contenidos registrados en los syllabus alrededor de unas temáticas generales y el programa específico de asignatura -el cual es mucho más puntual y aplicado-, que diseña y desarrolla cada profesor según sus propios intereses y formación. Más allá de los contenidos teóricos registrados en los syllabus, que cada profesor adapta y desarrolla en su respectivo programa de asignatura, los docentes cuentan que les interesa insistir en otros aprendizajes de los estudiantes, como la formación en sentido crítico frente a procesos de lectura y escritura, es decir, "aprender a leer y escribir críticamente". Esto permite considerar el espacio de formación teórica, como un terreno fértil para la abstracción y conceptualización alrededor de categorías clave en comunicación. Las prácticas de los profesores ponen en evidencia el debate que ya proponía Kant acerca de la formación como adiestramiento o como proceso para aprender a pensar, debate que queda abierto. Los syllabus de las denominadas teorías de o sobre la comunicación en los procesos de enseñanza aprendizaje de los programas de Bogotá, muestran que hay una apuesta institucional por darle una mirada particular a la comunicación, como comunicación para el desarrollo, periodismo, comunicación organizacional, comunicación audiovisual, entre otras, la cual se sintoniza con la filosofía de los programas.

Se encuentra un interés por ubicar al estudiante en el panorama de las teorías abordadas desde un solo tema, corriente o paradigma, hasta asignaturas que agrupan varios paradigmas o enfoques teóricos, y/o asignaturas que trabajan un concepto o categoría particular. Hay diversas entradas epistemológicas, entre las cuales se identifican la comunicación como ciencia, disciplina, campo de estudios u oficio. Además, se establece que las miradas desde las cuales se aborda la enseñanza de las teorías van desde el estudio cronológico, hasta ejes problémicos, pasando por el estudio de escuelas, modelos o relaciones interdisciplinarias, con el propósito de ubicar al estudiante en un panorama histórico que indique las teorías fundacionales y los nuevos debates de la comunicación a partir de la crisis de las ciencias sociales.

Vale la pena identificar que hay asignaturas que abordan temas muy específicos, como razón sensible, conflicto, acción colectiva, entre otras. Igualmente, se determina un núcleo de asignaturas cuyos contenidos y abordajes provienen de otras disciplinas distintas a la comunicación, pero que buscan enfatizar su carácter transdisciplinario. Algunos ejemplos son: economía política de la comunicación, proyecto experimental sobre 
razón sensible, estéticas y poéticas de la comunicación, sociología de la comunicación, y comunicación y conflicto.

Se puede concluir que hay una gran cantidad de enfoques, conceptos, categorías abordadas y articuladas a los diseños curriculares, oficios y aplicaciones que se hacen de la comunicación, con lo cual se entiende que este campo sostiene una tensión entre una riqueza y diversidad conceptual -inter y multidisciplinaria- entre campos del conocimiento de las ciencias humanas y las ciencias sociales.

A partir de los hallazgos parciales arrojados por los syllabus y por la herramienta Taller de devolución creativa - esta última concebida como una herramienta de metodología propia de la investigación participativa-, en relación con la bibliografía citada en los syllabus (figura 4), se observa que hay fuerte presencia de la escuela latinoamericana de la comunicación.

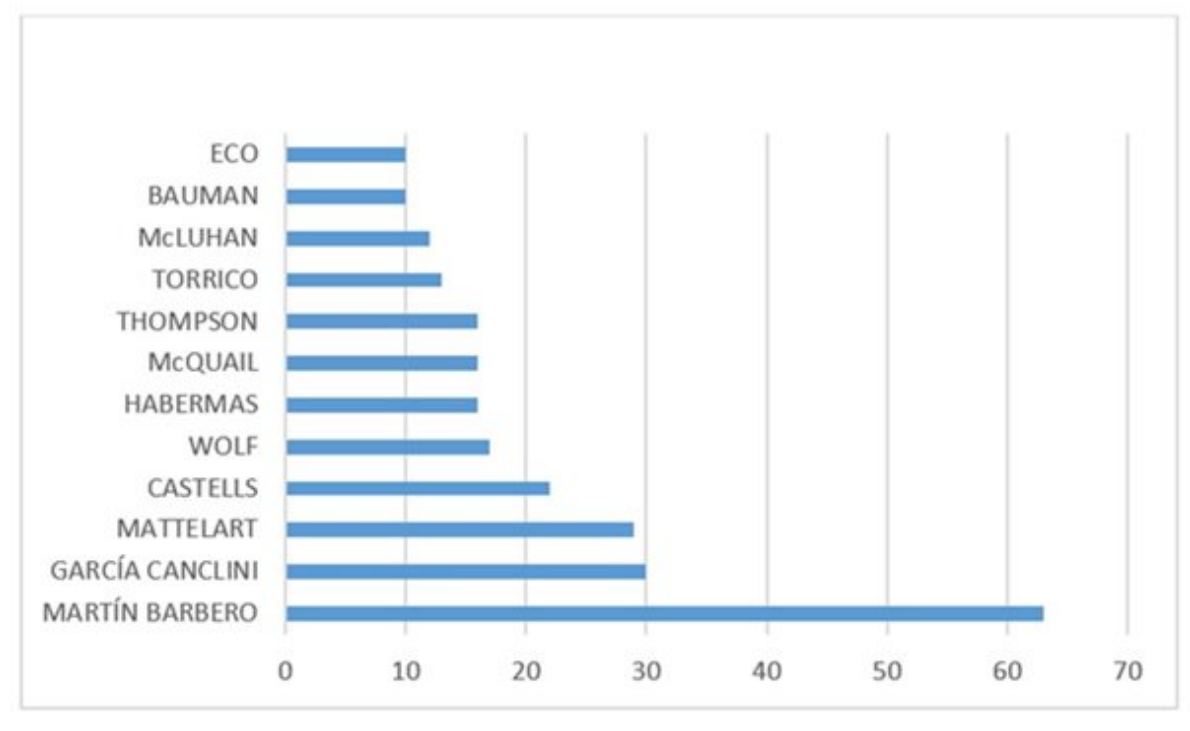

FIGURA 4

Bibliografía citada en los syllabus

Fuente: elaboración propia

Con relación a la comunicación-cultura y a la comunicación-desarrollo, los autores más citados en las bibliografías de los syllabus son Jesús Martín-Barbero y Néstor García Canclini -ambos por encima de autores europeos o anglosajones como Manuel Castells, Armand Mattelart, Mauro Wolf, Jürgen Habermas, Denis McQuail, J. P. Thompson-, seguidos por el autor boliviano Eric Torrico, Marshall McLuhan, Zigmunt Bauman y Umberto Eco. Cabe señalar que prácticamente en todas las referencias bibliográficas recogidas de los distintos programas, existe una precaria citación de autores y obras de investigadores colombianos, incluso cuando algunos de ellos son colegas de los docentes en las mismas instituciones. Lo anterior puede ser oportunidad para preguntarse por la producción intelectual de autores colombianos en este ámbito, y la debida socialización de trabajos académicos con la comunidad.

El entrecruce del análisis del syllabus con el análisis del taller de devolución creativa, posibilitó el diálogo con los profesores de teorías de la comunicación de las facultades de Comunicación Social de Bogotá, a quienes, en primera instancia, se les invitó a la socialización de los datos cuantitativos del análisis de los syllabus; y en una segunda instancia, a aportar desde cada experiencia a partir de la pregunta, ¿qué metodologías trabaja en su materia y qué busca lograr mediante estas? Los testimonios de los profesores resaltan las tendencias metodológicas en la enseñanza de dichas teorías. Afirman que es el syllabus el documento desde el que se diseña el programa de clase. En este se consigna todo, en la bibliografía incluyen generalmente autores canónicos, y las metodologías mayormente usadas son: clase magistral y debate en el aula. Así mismo, se privilegia el texto para que los estudiantes identifiquen el diálogo entre los autores y así logren comprender los fundamentos de cada uno y la diferenciación con los otros autores. También señalan el 
uso del cine y otras narrativas para reflexionar a partir de la conversación entre teoría y experiencias sociales de la comunicación. De igual manera, hacen uso de investigaciones de autores contundentes de la comunicación, a partir de la herencia teórica de América Latina, con el fin de hacer una apuesta por la comunicación como una práctica que genere procesos de transformación para pensar en categorías fuertes del campo. Dicen que es a partir de problemas de la comunicación que se abordan teóricamente los problemas contemporáneos del campo. Algunos profesores realizan actividades extra académicas, mesas de discusión con invitados y se plantea cumplir metas en torno a actividades que apoyan la formación integral de los estudiantes.

Los profesores destacan que se proponen propiciar aprendizajes en relación con la vida. Para esto, plantean en clase preguntas sobre la comunicación para llegar a definiciones construidas de forma colectiva: el foro colaborativo, el debate, la comprensión e interpretación colectiva, relatorías en las que se privilegia el texto desde el cual se confronta al estudiante con una pregunta por la vida diaria, el ejercicio de lectura, la escritura y la oralidad para optar por una redacción, y la sintaxis y la construcción adecuada en el discurso oral, son algunas de las prácticas que se realizan en clase. "Cabe anotar que las maneras de asumir la investigación, los énfasis y los objetos de estudio en comunicación han sido modificados en una serie de movimientos que determinan las prácticas metodológicas" (García Corredor y Rodríguez Bohórquez, 2011, p. 300), y componen un campo en construcción, como lo refiere Pereira (2005). El ejercicio que aquí se condensa contiene técnicas mixtas que permitieron recoger datos que resultan visionarios para entender que la enseñanza de las teorías de la comunicación no se inscribe en un único paradigma teórico, sino que, por el contrario, abarca un abanico complejo de las diferentes epistemes de la comunicación. En adición, permite identificar a la comunicación como un proceso más que como un saber objetivable, que se evidencia en la experiencia de aula que propicia el diálogo entre autores, teorías y otras narrativas. De igual manera, se encontró que los docentes acuden de manera indistinta a más de un enfoque metodológico al interior de su clase.

Para identificar los enfoques pedagógicos, la mirada a los syllabus se hace a partir de los objetivos, las estrategias pedagógicas y la evaluación, en relación con los enfoques pedagógicos tradicional, conductista, sociocultural y constructivista. Se encuentra, entonces, la siguiente tendencia (tabla 2).

TABLA 2

Tendencia enfoques pedagógicos

\begin{tabular}{ll}
\hline Tendencia de enfoques pedagógicos a partir de la revisión de los syllabus & Porcentaje \\
\hline Sociocultural (enfoque pedagógico - objetivo) & $52,1 \%$ \\
\hline Tradicional/constructivista (estrategias pedagógicas) & $23,3 \%$ \\
\hline No presenta aspectos a evaluar (evaluación) & $35,4 \%$ \\
\hline
\end{tabular}

Fuente: elaboración propia

Se estableció que los enfoques pedagógicos no se constituyen en estructuras rígidas para los profesores de teorías de la comunicación en su práctica pedagógica. Como se puede observar en la tabla, la tendencia manifiesta en los syllabus es el enfoque sociocultural (52,1\%). Este enfoque se caracteriza por propiciar procesos de aprendizaje mediante prácticas pedagógicas que fortalecen la construcción de conocimiento en contexto.

Las estrategias pedagógicas tradicional y constructivista $(23,3 \%)$ que aparecen como tendencia en los syllabus constituyen una combinación que alude a prácticas pedagógicas que privilegian, tanto el texto, como el proceso de aprendizaje. Esto se hace evidente en los testimonios de los profesores que, a la vez que se declaran como tradicionales en sus estrategias pedagógicas, privilegian lo magistral y buscan un propósito que da prioridad al proceso como "lograr una reflexión crítica complementada con un debate. Confrontar al estudiante con una pregunta por la vida diaria” (O. Arbeláez, comunicación personal, mayo 2015). 
De igual manera, se observa que hay una fuerte presencia de la escuela latinoamericana de la comunicación. Aunque en los syllabus la tendencia es no reportar aspectos a evaluar $(35,4 \%)$, durante los testimonios en el ejercicio cualitativo, la tendencia es a evaluar la teoría de manera tradicional, combinando esos visos conductistas con elementos del enfoque constructivista y sociocultural. Es decir, los profesores manifiestan que la memoria, la escritura y la sintaxis correcta, la oralidad, la comprensión de lectura, la reflexión crítica, la resolución de problemas y la intervención del entorno, no solamente facilitan la apropiación de contenidos, sino que también constituyen herramientas para el desempeño académico de los estudiantes.

De manera más específica, en los objetivos de las asignaturas prima el enfoque sociocultural, con un $52,8 \%$, y el tradicional, con un $22,2 \%$. En las estrategias pedagógicas prevalecen los enfoques tradicional y constructivista, con un 26,9\%; y es importante subrayar que, en relación con este aspecto, el 22,2\% de los syllabus no reportan estrategias pedagógicas. En los procesos y aspectos a evaluar, prima la tendencia sociocultural, con el 13\%, mientras que el 35\% no reporta aspectos ni enfoques de evaluación, lo cual es un indicio de su desactualización e incompletud como parte de la oferta pública de los contenidos que hacen los programas.

La noción de competencias, tal como está expresada en los syllabus, no permite identificar un concepto claro respecto de estas, a la vez que se presentan como un listado descriptivo, aislado de las metodologías, las formas de evaluar, los objetivos y los contenidos. Sin embargo, en el taller de devolución creativa se pudo identificar que los profesores de teorías buscan lograr en sus alumnos lo que el Ministerio de Educación Nacional de Colombia (MEN) sugiere al definir competencias como: "los conocimientos, habilidades y destrezas que desarrolla una persona para comprender, transformar y participar en el mundo en el que vive. La competencia no es una condición estática, sino que es un elemento dinámico que está en continuo desarrollo. Puede generar, potenciar apoyar y promover el conocimiento" (MEN, 2006).

El syllabus es un documento institucional que direcciona el desarrollo de los programas académicos, es un reflejo de lo que institucionalmente se busca con la asignatura en cuanto a contenidos, metodologías y bibliografías. Los resultados a partir del análisis de la encuesta virtual a los profesores de los distintos programas seleccionados para la investigación permitieron obtener 43 respuestas sobre un total de 62 profesores dedicados a la enseñanza de teorías de la comunicación (figura 5). Se infiere que la cátedra en los programas de comunicación en Bogotá, su representatividad y su distribución por géneros está, en su mayoría, a cargo de hombres.

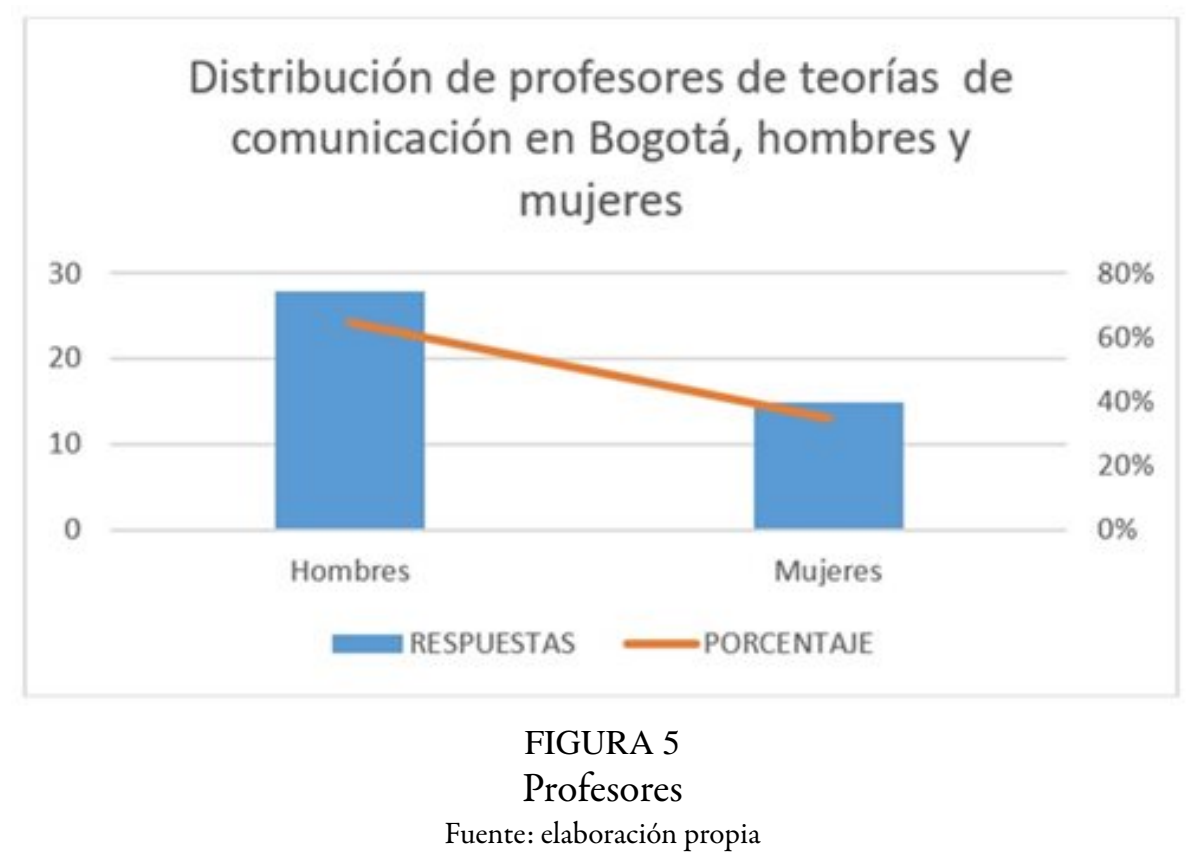


A partir de la muestra establecida sobre el perfil de profesores, se encuentra que un 54\% tiene formación básica de pregrado en Comunicación Social-Periodismo, con una edad promedio entre los 36 y los 45 años. El $70 \%$ concentra su trabajo en una sola universidad; mientras que un $23 \%$, en dos universidades.

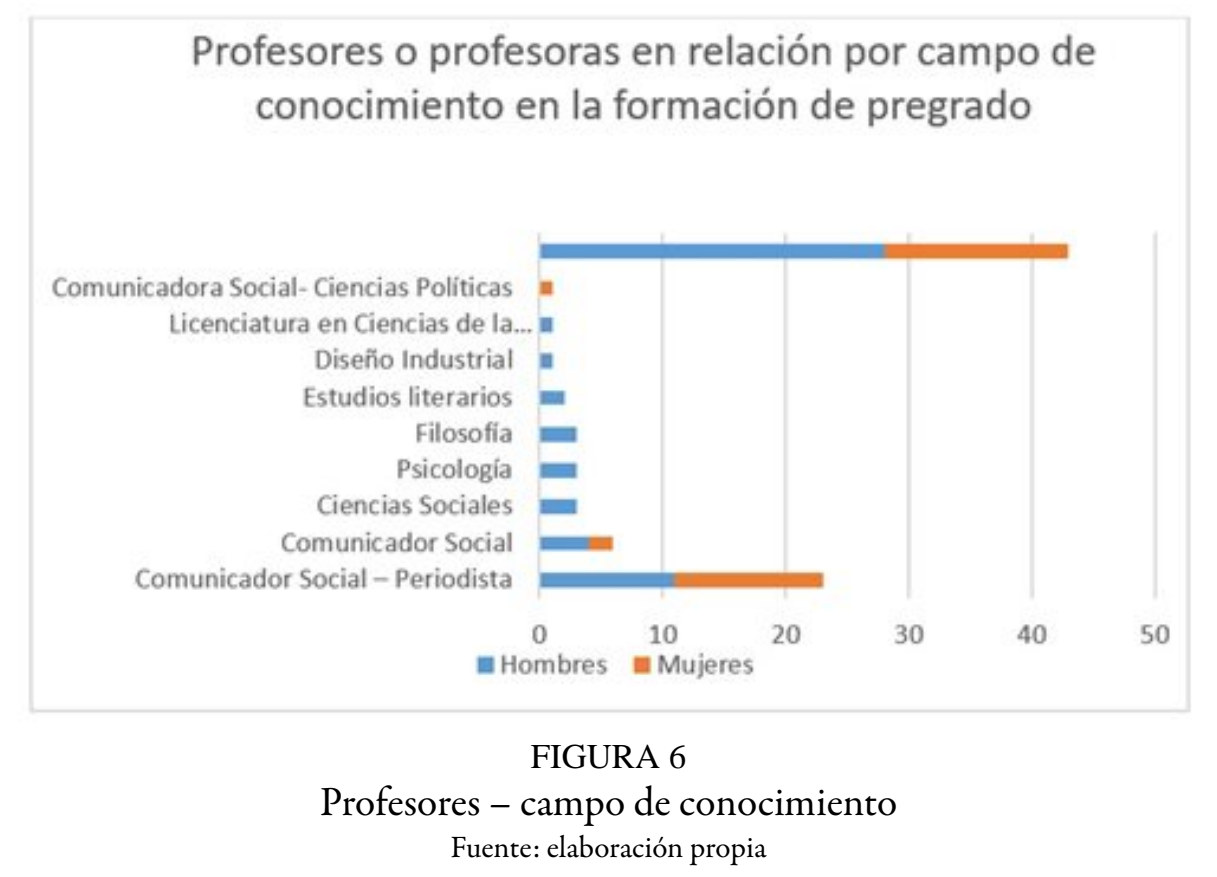

Se encontró que un $70 \%$ del total es de tiempo completo; y el $25 \%$, de medio tiempo, es decir que la muestra cuenta con un $5 \%$ de profesores de cátedra. Un $51 \%$ de los docentes de teorías lleva 10 años o más dedicado a la docencia de estas asignaturas; y un 40\%, entre tres y 10 años. El 93\% dice haber participado en proyectos de investigación sobre comunicación. Un $65 \%$ ha investigado sobre epistemología, teorías y/o métodos de la comunicación. Un porcentaje significativo, del 69\%, tiene al menos una publicación relacionada con el tema de teorías, un $25 \%$ ha publicado al menos un artículo en revista académica, un $24 \%$ ha participado con ponencia en eventos académicos, un $13 \%$ ha escrito capítulo en libro, y apenas un $3 \%$ ha publicado un libro relacionado con el tema de epistemología, teorías y/o métodos de la comunicación. De los 43 profesores reportados, 23 tienen como formación básica pregrado en Comunicación Social-Periodismo (figura 6).

De lo anterior puede inferirse que, en general, aunque los docentes de teorías de la comunicación en su mayoría tienen formación profesional en comunicación, investigan, escriben y publican muy poco sobre su práctica docente, sobre la epistemología de este campo de conocimiento y sobre los contenidos que enseña. Los enfoques pedagógicos son diversos, pero en líneas generales se ajustan a las políticas institucionales, sin perder la impronta de libertad de cátedra que se promueve al interior de las universidades. Los enfoques teóricos y metodológicos que se encuentran en los syllabus, y que reportan los profesores de comunicación, son una primera entrada descriptiva del proceso de enseñanza-aprendizaje de las teorías de comunicación en Bogotá. Así, los hallazgos aquí expresados en datos cualitativos y cuantitativos resultan ser un abrebocas para continuar con el abordaje investigativo en relación con el otro actor del proceso: el estudiante.

Así, los programas de comunicación en Bogotá incluyen asignaturas de teorías ofrecidas por profesores, en su mayoría, de comunicación, pero que tienen una importante pluralidad y diversidad en enfoques teóricos y metodológicos, orientados según los lineamientos de los proyectos institucionales, así como también a las preferencias teóricas de los profesores. 
TABLA 3

Metodologías de enseñanza de las teorías de comunicación.

Asignaturas analizadas a partir del syllabus: 108

\begin{tabular}{ll}
\hline Tipos de asignaturas & Tendencias entre los objetivos, las pedagogías y la evaluación \\
\hline $\begin{array}{l}\text { Asignaturas aplicadas a } \\
\text { las profesiones: }\end{array}$ & $\begin{array}{l}\text { La tendencia en los syllabus es: } 22 / 41 \text { son objetivos con enfoque } \\
\text { sociocultural en tanto privilegian el aprendizaje en contexto; } 12 / 41 \text {, con } \\
\text { estrategias pedagógicas tradicional y constructivista, combinación que alude } \\
\text { a prácticas pedagógicas que privilegian tanto el texto como el proceso de } \\
\text { aprendizaje, y } 17 / 41 \text { no presentan aspectos a evaluar. }\end{array}$ \\
\hline $\begin{array}{l}\text { Asignaturas específicas } \\
\text { sobre la comunicación: }\end{array}$ & $\begin{array}{l}\text { La tendencia en los syllabus es: } 13 / 28 \text { son objetivos con enfoque } \\
\text { sociocultural en tanto privilegian el aprendizaje en contexto; } 6 / 28, \text { con } \\
\text { estrategias pedagógicas tradicional y constructivista, combinación que alude } \\
\text { a prácticas pedagógicas que privilegian tanto el texto como el proceso de } \\
\text { aprendizaje, y } 11 / 28 \text { no presentan aspectos a evaluar. }\end{array}$ \\
\hline $\begin{array}{l}\text { Asignaturas } \\
\text { historiográficas: }\end{array}$ & $\begin{array}{l}\text { La tendencia en los syllabus es: } 22 / 39 \text { son objetivos con enfoque } \\
\text { sociocultural en tanto privilegian el aprendizaje en contexto; } 11 / 39, \text { con } \\
\text { estrategias pedagógicas tradicional y constructivista, combinación que alude } \\
\text { a prácticas pedagógicas que privilegian tanto el texto como el proceso de } \\
\text { aprendizaje, y } 10 / 39 \text { no presentan aspectos a evaluar. }\end{array}$ \\
\hline
\end{tabular}

- $57 / 108$ son objetivos con enfoque sociocultural en tanto privilegian el aprendizaje en contexto ( $24 / 108$ con enfoque tradicional en los objetivos).

- $29 / 108$ son estrategias pedagógicas tradicional y constructivista, combinación que alude a prácticas pedagógicas que privilegian tanto el texto como el proceso de aprendizaje (24/108 no presentan estrategias pedagógicas).

- $38 / 108$ no presentan aspectos a evaluar ( $14 / 108$ con tendencia sociocultural en los aspectos a evaluar).

Fuente: elaboración propia

\section{Referencias}

Alsina, R. (2001). Teorías de la comunicación. Ámbitos, métodos y perspectivas. Barcelona: UAB.

Berelson, B. (2004). The State of Communication Research. En J. Peters y P. Simonson (eds.), Mass Communication and Social Thought (pp. 440-445). Maryland, Estados Unidos: Rowman \& Littlefield.

de Moragas, M. (2011). Interpretar la comunicación. Estudios sobre medios en América y Europa. Barcelona: Gedisa.

de Zubiría, J. (2008). Los modelos pedagógicos. Hacia una Pedagogía dialogante. Bogotá: Magisterio.

Fals Borda, O. (2008). La investigación acción en convergencias disciplinares. Recuperado de http://historiactualdos.bl ogspot.com.ar/2008/11/la-investigacin-accin-en-convergencias.html.

Flórez, R. (2008). Pedagogías del Conocimiento. Bogotá: Mcgraw Hill.

Fuentes Navarro, R., y Vasallo de Lopes, I. (comps.). (2001). Comunicación, campo y objeto de estudio. Guadalajara: Iteso.

Galindo, J. (2008). Comunicación, ciencia e historia. Fuentes cientificas históricas hacia una comunicología posible. Madrid: McGraW Hill Interamericana.

García Corredor, C. P., y Rodríguez Bohórquez, L. F. (2011). Sobre profesiones, prácticas y oficios de la comunicación. Signo y Pensamiento, 31(59), 298-306.

Kant, E. (s. f.). Pedagogía. Recuperado de https://www.ddooss.org/articulos/textos/kant_pedagogia.pdf. 
Martín Serrano, M. (2006). Para qué sirve estudiar Teoría de la Comunicación. Contratexto, (14), 41-48.

Martino, L. (2007). Teorias da Comunicaçao: mutias ou poucas. Sao Paulo: Atelie.

Mattelart, A. (1997). Historia de las teorías de comunicación. Barcelona: Paidós.

Maxwell, J. (2004). Reemergent scientism, postmodernism, and dialogue across diferences. Revista Qualitative Inquiry, (10), 35-41.

Ministerio de Educación Nacional de Colombia (MEN). (2006). Guía No. 3. Manual de evaluación del desempeño de docentes y directivos docentes. Bogotá: MEN. Recuperado de https://www.mineducacion.gov.co/1759/w3-artic le-81030.html.

Pereira, J. M. (2005). La comunicación: un campo de conocimiento en construcción Reflexiones sobre la comunicación social en Colombia. Investigación \& Desarrollo, 13(2), 412-441.

Rizo, M. (2012). Las teorías de la comunicación en la construcción del campo académico de la comunicación: apuntes históricos, reflexiones epistemológicas y retos pedagógicos. Lima: Congreso de Felafacs.

Saintout, F. (2003). Abrir la comunicación. Tradición y movimiento en el campo académico. La Plata: Facultad de Periodismo y Comunicación Social Universidad de la Plata.

Torrico, E. (2004). Abordajes y periodos de la teoría de la comunicación. Buenos Aires: Norma.

Torrico, E. (2010). Comunicación: de las matrices los enfoques. Quito: Ciespal.

Vidales, C. (2010). Semiótica y Teoría de la Comunicación. Tomo I. Monterrey: Colegio de Estudios Científicos y Tecnológicos del Estado de Nuevo León.

Vidales Gonzáles, C. (2011). El relativismo teórico en comunicación. Entre la comunicación como principio explicativo y la comunicación como disciplina práctica. Comunicación y Sociedad, (16), 11-45.

\section{Notas}

* Artículo de investigación.

i Las siguientes universidades: Sabana, Central, Externado de Colombia, El Rosario, Politécnico Grancolombiano, Uniminuto, Sergio Arboleda, INPAHU, Jorge Tadeo Lozano, Santo Tomás, UNAD, Cooperativa, Unipanamericana, Los Libertadores, San Alfonso Ligorio y la Pontificia Universidad Javeriana.

\section{Licencia Creative Commons CC BY 4.0}

Cómo citar este artículo: García Corredor, C. P., Castellanos Barbosa, Á. M., y Pereira González, J. M. (2019). La enseñanza de la comunicación: una mirada a los enfoques teóricos y metodológicos de profesores y programas de teorías de comunicación en Bogotá. Signo y Pensamiento, 38(75). https://doi.org/10.11144/ Javeriana.syp38-75.ecme 